SUBJECT AREAS:

NANOWIRES

SURFACE PATTERNING

LITHOGRAPHY

Received

1 September 2014

Accepted

24 February 2015

Published

18 March 2015

Correspondence and requests for materials should be addressed to

N.M. (nassirmojarad@ethz.ch) or Y.E. (Yasin.Ekinci@ psi.ch)

* Current address: Nanotechnology Group, ETH Zurich, Säumerstrasse 4, 8803 Rüschlikon, Switzerland.

\title{
Beyond EUV lithography: a comparative study of efficient photoresists' performance
}

\author{
Nassir Mojarad*, Jens Gobrecht \& Yasin Ekinci
}

Laboratory for Micro- and Nanotechnology, Paul Scherrer Institute, 5232 Villigen, Switzerland.

Extreme ultraviolet (EUV) lithography at $13.5 \mathrm{~nm}$ is the main candidate for patterning integrated circuits and reaching sub-10-nm resolution within the next decade. Should photon-based lithography still be used for patterning smaller feature sizes, beyond EUV (BEUV) lithography at 6.x nm wavelength is an option that could potentially meet the rigid demands of the semiconductor industry. We demonstrate simultaneous characterization of the resolution, line-edge roughness, and sensitivity of distinct photoresists at BEUV and compare their properties when exposed to EUV under the same conditions. By using interference lithography at these wavelengths, we show the possibility for patterning beyond $22 \mathrm{~nm}$ resolution and characterize the impact of using higher energy photons on the line-edge roughness and exposure latitude. We observe high sensitivity of the photoresist performance on its chemical content and compare their overall performance using the Z-parameter criterion. Interestingly, inorganic photoresists have much better performance at BEUV, while organic chemically-amplified photoresists would need serious adaptations for being used at such wavelength. Our results have immediate implications for deeper understanding of the radiation chemistry of novel photoresists at the EUV and soft X-ray spectra.

M oore's law indicates that the number of transistors in integrated circuits should double about every two years and it has been the paradigm of the semiconductor industry for about five decades. Downscaling feature sizes allows for faster processing with more power efficiency at a lower $\operatorname{cost}^{1,2}$, but physical and practical barriers hinder this progress as they have reached nanometer-size dimensions ${ }^{3}$. Photolithography has been the only method that meets large-scale patterning throughput for the semiconductor industry but comes with its intrinsic limitations. In optical projection lithography systems the resolution is limited by diffraction of light, and therefore, the wavelength used for the semiconductor industry has over time experienced many transitions from visible to deep ultraviolet (DUV) of $\lambda=193 \mathrm{~nm}$, which is currently the dominant patterning wavelength. As a continuation of this trend, extreme ultraviolet (EUV) wavelength of $\lambda=13.5 \mathrm{~nm}(92 \mathrm{eV})$ is the major candidate for the next-generation lithography. In the last decade, significant investment has been made in the development of EUV lithography (EUVL) for mass production of integrated circuits. Several demo and preproduction EUVL scanners have been successfully installed and the third generation is expected to meet highvolume chip manufacturing for the technology nodes with below $22 \mathrm{~nm}$ half-pitch (HP) ${ }^{4}$. In less than a decade EUVL is expected to be optimized to its best for sub-10-nm resolution manufacturing, before the patterning resolution also reaches the limit set by light diffraction. The question of how far can photolithography be used for patterning integrated circuits was raised when immersion lithography at DUV was reaching its limits and even then there were serious competitors for EUVL such as electron beam (e-beam), directed self assembly, and nanoimprint lithography ${ }^{5}$. The possibility of the semiconductor industry still using photolithography after EUVL reaches its resolution limits highly relies on the successful development of the relevant technologies such as optics-related elements and photoresist materials. Functional photoresists should provide the RLS characteristics, that is, resolution (R), line-edge roughness (LER), and sensitivity (S). A major issue to set for post-EUV photolithography is the wavelength of choice. Using $\lambda \approx 1 \mathrm{~nm}$ drew much attention more than two decades ago but due to various practical, physical, and chemical barriers failed to be used for sub-100 nm patterning ${ }^{6}$. Using $\lambda=6 . \mathrm{xmm}$, coined as Beyond EUV (BEUV), is the most prominent candidate for patterning even smaller features using photons ${ }^{1,2}$. This specific wavelength range is proposed by industry because it could potentially meet requirements of the light source, as well as reflective and imaging optics ${ }^{7}$. The leap from DUV to EUV is a huge technological challenge due to several reasons such as all-in-vacuum operation, reflective projection optics, 
effective light-source, and material response of photoresists at substantially different photon energies. Nevertheless, once EUV technology is adapted for high-volume manufacturing, the transition from EUV to BEUV will be relatively straightforward. From the photoresist point-of-view, the radiation chemistry reveals similar properties at the two wavelengths, although the optical properties of materials rapidly change in the EUV and soft X-ray regime, due to the presence of sharp atomic absorption edges ${ }^{8}$.

In recent years, extensive research has been devoted to developing efficient BEUV instrumentation, mainly light sources ${ }^{9}$ and reflective optics $^{10-13}$, demonstrating the feasibility of BEUV lithography. From the photoresist side, also, the response to BEUV illumination has been a subject of recent studies. Oyama et al. made pioneering studies on sensitivities of several photoresists at EUV, BEUV, and shorter wavelengths ${ }^{14,15}$. In addition, Anderson et al. investigated the sensitivity of high-resolution photoresists and concluded that the relative sensitivities at the two wavelengths are due to their different optical absorption coefficients ${ }^{16}$. In order to consider BEUV lithography as an option for future lithography generations, it is also important to explore its patterning capabilities and demonstrate that providing photoresists satisfying stringent RLS requirements are feasible. Since lithography at BEUV is in its emergence phase, there is yet no effective lithography system integrated with all the needed components such as optics, light source, and masks. Interference lithography (IL) with diffraction gratings, on the other hand, enables a platform for evaluating patterning capabilities of photoresists at a broad spectral range because it provides a wavelength-independent aerial image ${ }^{17,18}$. As illustrated in Fig. 1a, to make line-space patterns, the collimated beam illuminates a mask holding a set of two parallel gratings with periodicity $P_{\mathrm{g}}$. The $1^{\text {st }}$-order diffracted beams overlap at a certain distance from the mask to form a periodic aerial image. By placing a photoresist-coated wafer there, a periodic line-space pattern is obtained with $\mathrm{HP}=P_{\mathrm{g}} / 4$. Moreover, thanks to the wavelength tunability of synchrotron sources and availability of high-resolution broadband masks ${ }^{19}$, efficient patterning and direct comparison of photoresists at different wavelengths becomes possible in a straightforward manner ${ }^{20}$. Here, we report on exploring BEUV lithography at $\lambda=6.5 \mathrm{~nm}$ by patterning various photoresists with different backbone chemistries at cutting-edge resolutions, evaluating their RLS parameters, and studying some lithography limitations and the involved processes. The lithography trade-off principle indicates that within a certain backbone chemistry, reducing one or two of the RLS components comes with the cost of losing at least one of the others $^{21}$. Therefore, studying RLS variations in these intrinsically different resists paves the way for understanding the appropriate chemistry for effective BEUV lithography.

\section{Results}

We tested the lithographic performance of three high-performance photoresists, at EUV and BEUV. Inpria XE15IB (IB) is a modern hafnium-based inorganic photoresist ${ }^{22}$, which has excellent sensitivity and has been demonstrated to be used for photolithography at highest resolutions ${ }^{23,24}$. The second photoresist we study is hydrogen silsesquioxane (HSQ), an inorganic photoresist with very high resolution but low sensitivity. It has been extensively used for e-beam and EUV lithography ${ }^{25}$, and its well-known chemistry makes it a good candidate for analyzing its response to high-energy photons and electrons. The third photoresist we study is an organic chemically amplified resist (CAR), which is among the highest resolution resists of this kind, and has an intermediate dose.

We examine patterning properties at the two wavelengths by using a broadband IL mask ${ }^{19}$ containing gratings with various periodicities, down to $P_{\mathrm{g}}=72 \mathrm{~nm}$, corresponding to $\mathrm{HP}=18 \mathrm{~nm}$ on wafer. Details of mask fabrication are described in Methods. For each wavelength, the gap between the mask and the wafer is adjusted to result in maximum overlap of the diffracted beams (Fig. 1a) and a dose scan is a

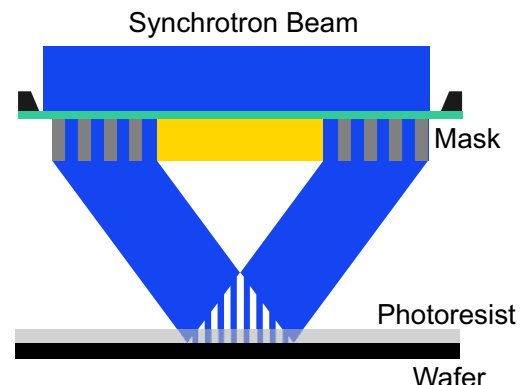

b

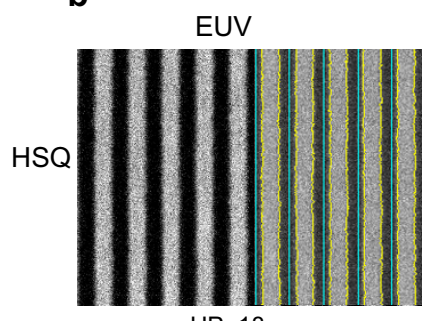

$\mathrm{HP}=18 \mathrm{~nm}$
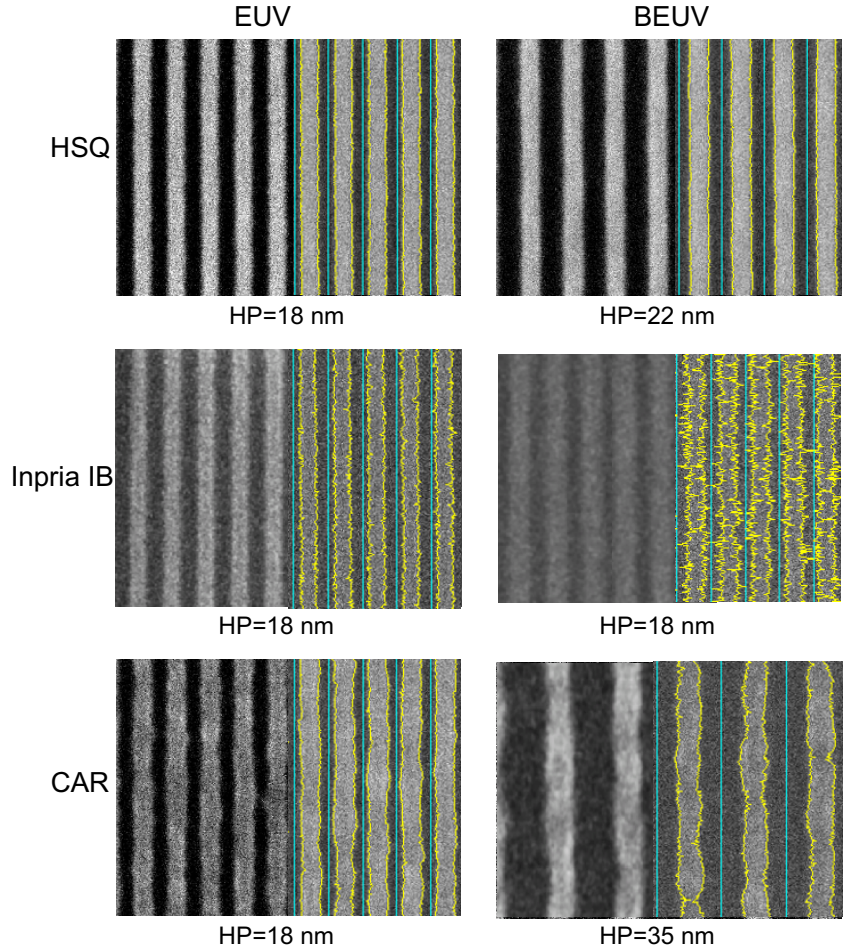

$\mathrm{HP}=22 \mathrm{~nm}$

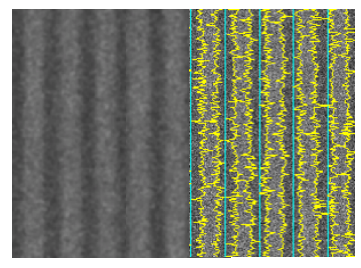

$\mathrm{HP}=18 \mathrm{~nm}$

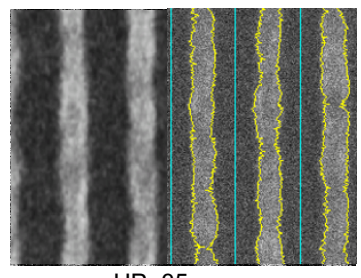

$\mathrm{HP}=35 \mathrm{~nm}$

Figure 1 Interference lithography and patterning line-space structures. (a) Schematic of interference lithography using a broadband mask for making line-space patterns. (b) Scanning-electron micrographs of Inpria IB (first row), HSQ (second row), and CAR (third row) at EUV (left column) and BEUV (right column). The half-pitch of each image is $\mathrm{HP}_{\text {min }}$ and is stated under it. Yellow traces show the analyzed line profile, overlaid on the corresponding lines.

performed on the same wafer. We note that in IL, depth-of-focus is not an issue since the aerial image is independent of the gap. Thus, our tool provides the same aerial image at different wavelengths and enables using the same mask and wafer at different wavelengths and therefore makes it possible to compare the patterning capabilities of photoresists at different wavelengths in a simple manner. Figure $1 \mathrm{~b}$ illustrates scanning-electron micrographs (SEMs) of the three photoresists patterned at both wavelengths with their minimum patternable feature size $\left(\mathrm{HP}_{\min }\right)$, where lines were distinctly separated throughout the patterned area and revealed no bridging or pinching. As it can be seen, the three photoresists have been patterned at EUV down to $\mathrm{HP}=18 \mathrm{~nm}$, while only the Inpria resist could provide such resolution at BEUV. HSQ patterns have very smooth edges at both wavelengths but the LER increases in the other two photoresists. Moreover, the fitted traces on the Inpria resist line edges clearly indicate that for this photoresist, patterning at BEUV noticeably increases the LER and reduces the patterning contrast. In the case of the CAR, the achieved $\mathrm{HP}_{\text {min }}$ is almost two times higher than that at EUV and the lines reveal clear necking. We point out that $\mathrm{HP}_{\min }=$ 
$18 \mathrm{~nm}$ is the smallest feature sized achievable with this mask and experiments are planned for determining more accurate resolution limits by making masks hosting gratings with smaller pitches.

The critical dimension (CD) and LER dose-dependence is illustrated in Fig. 2. The analysis of the CD and LER was done using a commercial software (SuMMIT ${ }^{\circledR}$ ) and LER values correspond to $3 \sigma$ deviation averaged over both walls of 20 lines. The horizontal axis has been calibrated to represent dose-on-wafer, $D_{\mathrm{w}}$ and not the commonly used dose-on-mask $D_{\mathrm{m}}$. This calibration allows comparison of the exact dose at the wafer and takes into account the mask diffraction efficiency difference at the two wavelengths. Details of the procedure are described in Methods. As it can be seen, since Inpria IB is a negative-tone resist, for all HPs CD monotonically increases by increasing $D_{\mathrm{w}}$, and remarkably, the exposure latitude (EL) drops at all HPs when moving from EUV to BEUV. The ratio of the ELs at BEUV to EUV, $Q_{\mathrm{EL}}$ is 0.25 for $\mathrm{HP}=35 \mathrm{~nm}$ and increases to $Q_{\mathrm{EL}} \approx$ 0.4 for $\mathrm{HP}=20$ and $22 \mathrm{~nm}$, yet at the $\mathrm{HP}=18 \mathrm{~nm}$ illuminated by BEUV, only patterns could be formed at one dose value. Within the same functional dose range, the dependence of the LER on $D_{\mathrm{w}}$ is illustrated in Fig. 2b. Clearly, the LER is lower at all HP values when exposed with EUV in comparison to BEUV. Whether rougher edges are solely because of the chemical formulation of the resist, or because of an intrinsic property of exposure at higher photon energies can be examined by comparing patterning performance of other photoresists. Figure 3 a demonstrates the average LER as a function of HP for all photoresists. In terms of LER, HSQ has a good performance at both wavelengths, and provides LER $<2 \mathrm{~nm}$, whereas for Inpria IB and the CAR, patterning at BEUV results in rougher edges. For HSQ, BEUV exposures provided $\mathrm{HP}_{\min }=22 \mathrm{~nm}$, while with EUV $\mathrm{HP}_{\text {min }}=18 \mathrm{~nm}$ was achieved, which confirms similar measurements that show $\mathrm{HP}_{\text {min }} \approx 15 \mathrm{~nm}^{26}$. In principle this resolution limit for HSQ might be improved by using another developer based on $\mathrm{NaOH}$ solution, commonly used for developing patterns with HP $<15 \mathrm{~nm}^{24,26}$. However, this improvement comes with the cost of losing sensitivity by a factor of 3 at EUV wavelength. The CAR we studied is among the highest-resolution resists and revealed down to $\mathrm{HP}_{\text {min }}=18 \mathrm{~nm}$ at EUV. However, the smallest feature size observed at BEUV was only $\mathrm{HP}_{\min }=35 \mathrm{~nm}$. In a previous study we demonstrated that another CAR, which has the EUV resolution limit of $\mathrm{HP}_{\text {min }}=22 \mathrm{~nm}$, can also be patterned with the same resolution at $\mathrm{BEUV}^{20}$. It could therefore be concluded that $35 \mathrm{~nm}$ is not the patterning resolution limit of organic CARs and that their performance is strongly dependent on their chemical composition and the incident photon energy.

The photoresist sensitivity is the next parameter that was addressed by making a dose-to-clear exposure. In this process a thin layer of spin-coated photoresist $(\sim 30 \mathrm{~nm})$ is exposed through an

a

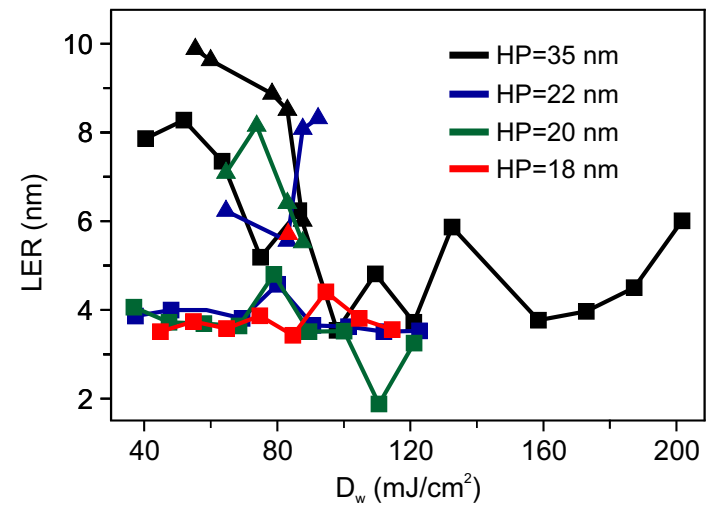

open frame at different dose values and after development the photoresist height is mapped as a function of $D_{\mathrm{w}}$. The $50 \%$ clearance of the fitted function, $D_{0}$, is regarded as the photoresist sensitivity. Although it is suggested that the absorbed dose per unit mass is a more general quantity for characterizing the sensitivity ${ }^{15}$, the above defined $D_{0}$ has also proven to be an accurate measure of the sensitivity in other similar studies ${ }^{16}$, without requiring the chemical and physical properties of the resists, and is thickness independent for our very thin $(<50 \mathrm{~nm})$ layers. Figure $3 \mathrm{~b}$ illustrates the normalized contrast curves of the three resists at the two wavelengths. By comparing the sensitivities at the two wavelengths (Table 1), it can be seen that HSQ is the only photoresist that requires less dose at BEUV than at EUV. Interestingly, for Inpria IB the sensitivities are quite comparable while for the CAR the BEUV sensitivity is noticeably worse. From the same curves, the photoresist contrast, $\gamma$, defined as $\gamma=1 / \log _{10}\left(D_{2} / D_{1}\right)$ is also extracted and listed in Table 1 . In this relation $D_{1}$ is the highest/lowest dose and $D_{2}$ is the lowest/highest dose at which a positive/negative-tone resist is $100 \%$ dissolved/ remained after exposure and development. The $\gamma$ quantity is conventionally used to evaluate different aspects of a resist performance. Going from EUV to BEUV, $\gamma$ values significantly decrease for Inpria $\mathrm{IB}$, and increases for HSQ and the CAR.

\section{Discussion}

In common photoresists, where the latent image formation is governed by processes such as molecular bond scission or crosslinking, the sensitivity of the resist is proportional to its optical absorption coefficient, and as a result it is expected that $D_{0} \propto l$, where $l$ is the resist attenuation length. We define $Q_{D}$ as the ratio of the measured $D_{0}$ at BEUV to EUV, and similarly $Q_{l}$ as the ratio of the theoretically calculated $l$ at the two wavelengths. Figure $3 c$ illustrates $Q_{D}$ (solid bars) and $Q_{l}$ (dashed bars) for the photoresists, as well as $Q_{l}$ values for the elements that compose them. The $l$ values were calculated based on the chemical formulations using reference ${ }^{27}$ and in the case of Inpria IB and the CAR, although the chemical formula is not disclosed, the companies provided us with the optical constants at the two wavelengths and $l$ was deduced accordingly. As mentioned earlier, HSQ has higher sensitivity at BEUV, which is attributed to the very high absorption of $\mathrm{Si}$ in its backbone chemistry ${ }^{14}$. An interesting feature of HSQ is that $Q_{D} \approx Q_{l}$, which indicates that the cross-linking rate of the matrix mainly depends on the absorption of its atoms. For a certain photoresist the amount of dose-to-clear depends on the number of absorbed photons as well as their energy. When a highenergy photon strikes a photoresist matrix, it loses energy mainly by creating secondary electrons (SEs), and these SEs scatter in the resist and further produce lower energy SEs until the SE energies are below the ionization energy. When comparing the SE generation using

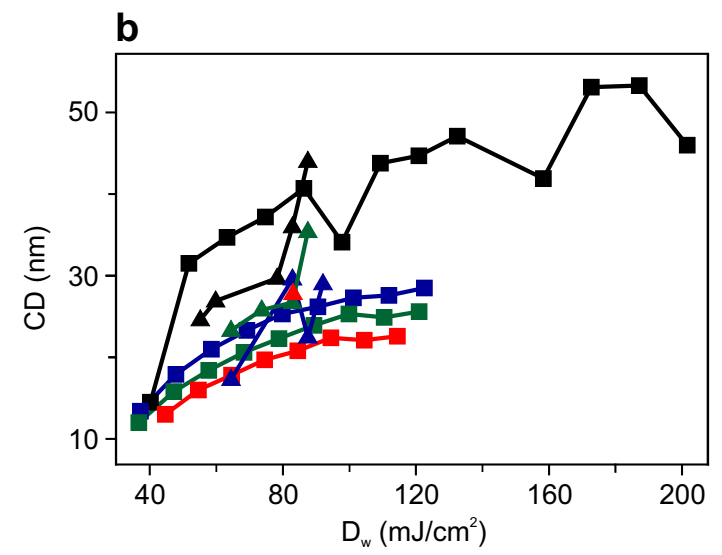

Figure $2 \mid$ Line-edge roughness and critical dimension of Inpria IB. (a) LER and (b) critical dimension as a function of dose-on-wafer for different halfpitch values, exposed at EUV (squares) and BEUV (triangles). 

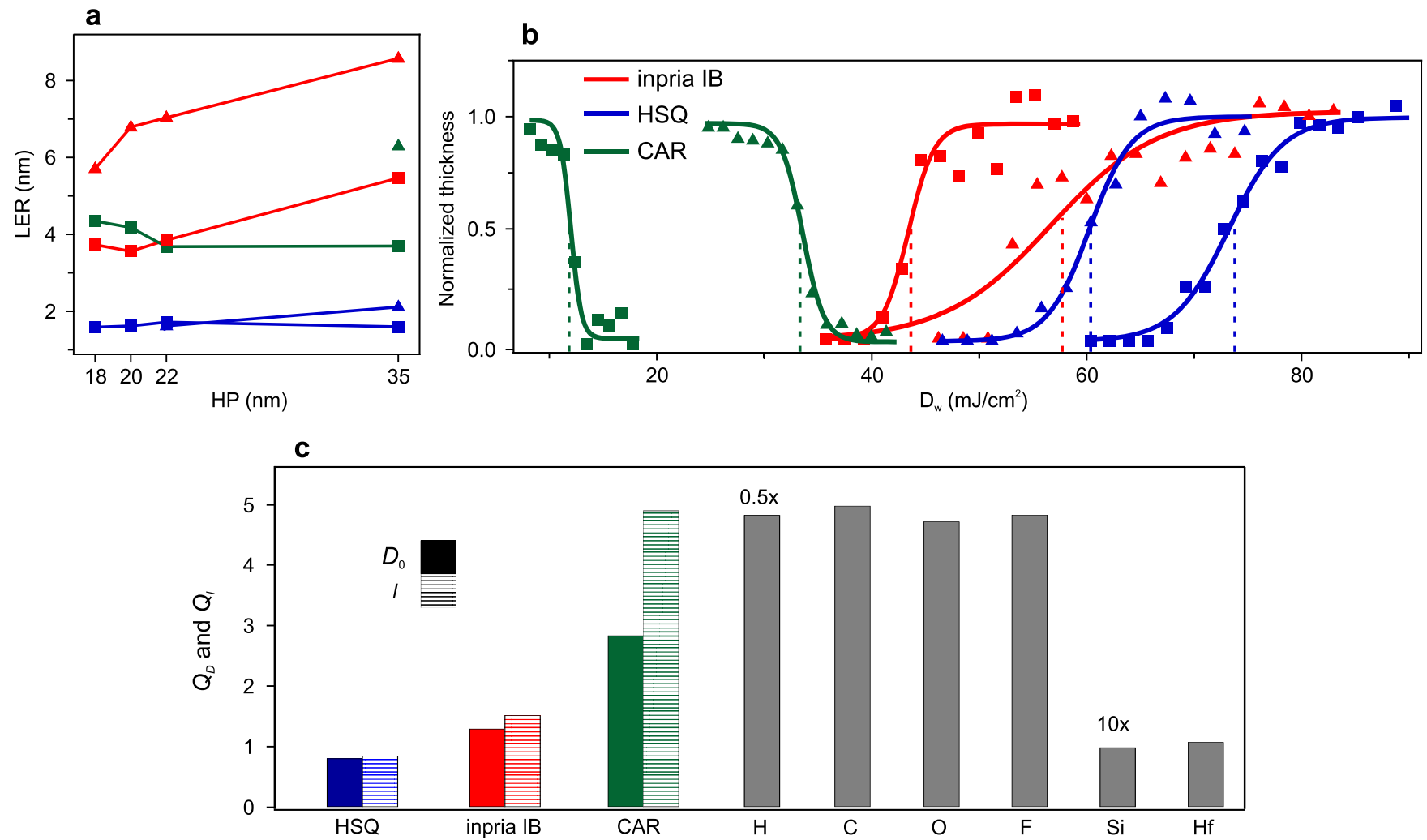

Figure 3 Resolution, line-edge roughness and sensitivity characterization of the three photoresists. (a) LER as a function of HP of Inpria IB (red), HSQ (blue), and CAR (green), exposed at EUV (squares) and BEUV (triangles). (b) Normalized thickness of the photoresists exposed through an open frame as a function of $D_{\mathrm{w}}$. Dashed line indicate $D_{0}$ values for each case. (c) Measured $Q_{\mathrm{D}}$ (solid) and calculated $Q_{l}$ (dashed) values of the photoresists. Gray bars represent calculated $Q_{l}$ values for elements present in the chemical formula of the photoresists.

EUV and BEUV, the later has a higher energy and consequently produces a larger number of SEs per absorbed photon. On the other hand EUV photons have lower energy and as a result, for a certain amount of dose more photons exist to start the SE generation process. According to Fig. 3c, this balance results in $D_{0} \propto l$ for HSQ and Inpria IB, while for the CAR the required dose at $\mathrm{BEUV}$ is significantly less than what is expected if the dynamics were solely governed by the absorption of the host matrix. Therefore, within the energy range that we study, exposing CARs using higher energy photons increases the chemical amplification effect, and reduces the required dose.

The effect of the resist contrast also plays a noticeable role in the final pattern quality. Quantitative comparison of this effect can be made by considering the $\gamma$ values (Table 1), where larger $\gamma$ s represent higher contrast in the resists and consequently higher pattern quality. In the case of HSQ, these values are very similar and in fact slightly larger for BEUV than EUV. In the case of Inpria IB, $\gamma$ is noticeably less at the higher photon energy, which directly reveals itself in the worse patterning contrast, that can be seen in Fig. 1b. In the case of the CAR, although $\gamma$ is larger for the BEUV exposure, the dynamics of the interaction of higher energy photons and the generated SEs with the resist, together with the acid diffusion process does not allow high resolution patterning at the level that is possible at EUV. From the technological viewpoint, an important issue to address is the limitations that are held for the chemical compositions of BEUV resists. Most present low-dose EUV resists, including our studied CAR, owe their sensitivity to chemical amplification and the high photon absorption of their organic backbone chemistry. Indeed C, O, and $\mathrm{F}$ are common elements of many efficient EUV photoresists, and as it can be seen in Fig. 3c, their absorption is about 5 times less at BEUV. As a result, the issue of sensitivity will be a barrier for BEUV lithography of organic photoresists. On the other hand, after a rapid growth in developing organic CARs, in recent years there has been a raising trend to overcome its limitations using inorganic photore$\operatorname{sists}^{22,28,29}$, which could be a promise for their implementation also at BEUV.

Among the photoresist we studied Inpria IB is the only one that could be successfully patterned at BEUV with $\mathrm{HP}<22 \mathrm{~nm}$. Beside this high resolution it provides, the LER is noticeably higher in comparison to EUV patterning. To find the reason for this higher LER, we take a further step of analyzing it into its main components. LER sources could be divided into three main categories, namely that associated with shot noise $\left(\mathrm{LER}_{\mathrm{SN}}\right)$, the photoresist material $\left(\mathrm{LER}_{\text {mat }}\right)$, and the process $\left(\mathrm{LER}_{\text {proc }}\right)^{30}$, that add up quadratically such

Table $1 \mid D_{0}$ and $\gamma$ values of the three photoresists at EUV and BEUV wavelengths

\begin{tabular}{lccrr} 
& \multicolumn{2}{c}{$D_{0}\left(\mathrm{~m} J / \mathrm{cm}^{2}\right)$} & \multicolumn{2}{c}{$\gamma$} \\
\cline { 2 - 3 } \cline { 4 - 5 } & EUV & BEUV & EUV & BEUV \\
\hline HSQ & 73.8 & 61.0 & 11.76 & 11.79 \\
Inpria IB & 43.3 & 56.7 & 14.08 & 4.84 \\
CAR & 11.6 & 33.2 & 7.49 & 11.17 \\
\hline
\end{tabular}


that the total LER can be written as follows:

$$
\mathrm{LER}^{2}=\mathrm{LER}_{\mathrm{SN}}^{2}+\mathrm{LER}_{\mathrm{mat}}^{2}+\mathrm{LER}_{\text {proc }}^{2} .
$$

Shot noise is caused by the random fluctuations of the limited number of absorbed photons, which follows Poisson statistics, and reveals itself in the patterned lines as variations at the edges of the exposed structures. Assuming that the resist absorbs a dose of $D_{\mathrm{a}}$, corresponding to $N_{\mathrm{a}}$ photons at wavelength $\lambda$, it is commonly known that $\mathrm{LER}_{\mathrm{SN}} \propto 1 / \sqrt{ } N_{\mathrm{a}}{ }^{31,32}$. Moreover, since $N_{\mathrm{a}}=D_{\mathrm{a}} \lambda / h c$, where $h$ and $c$ are accordingly the Planck's constant and the speed of light in vacuum, it could be derived that:

$$
\mathrm{LER}_{\mathrm{SN}} \propto 1 / \sqrt{ } D_{\mathrm{a}} \lambda,
$$

which implies that $\mathrm{LER}_{\mathrm{SN}}$ increases at shorter wavelengths. In a hypothetical resist which has identical $D_{\mathrm{a}}$ at both EUV and BEUV, $Q_{\mathrm{LER}}=1 / \sqrt{ } Q_{\lambda}=1.44$, where $Q_{\mathrm{LER}}$ and $Q_{\lambda}$ are respectively the ratios of the LER and $\lambda$ at BEUV to EUV. Although real resists could not be treated in that way, we can still investigate whether shot noise alone can justify the large value of $Q_{\mathrm{LER}}$ in Inpria or not. According to Fig. 3a, for $\mathrm{HP}=22 \mathrm{~nm}$ we experimentally measure $\mathrm{Q}_{\mathrm{LER}}=1.83$, while Fig. 3c indicates $Q_{D}=1.31$ and consequently, according to Eq. $2, Q_{\text {LER }}$ only caused by shot noise is $1 / \sqrt{ } Q_{D} Q_{\lambda}=1.26$. This means that the pure shot noise cannot justify the discrepancy of LER at the two wavelengths, and $\mathrm{LER}_{\text {mat }}$ and $\mathrm{LER}_{\text {proc }}$ also have a noticeable effect. Among the process factors, what significantly changes the patterning quality is flare cause by defects on the masks and grating roughness. There is no direct study of the effect of grating roughness on the final aerial image in an IL layout. However, based on the fact that the induced speckles cause by mask roughness directly influence the $\mathrm{LER}_{\text {proc }}{ }^{33}$, reducing the wavelength increases the $\mathrm{LER}_{\text {proc }}$. Regarding $\mathrm{LER}_{\text {mat }}$, for a certain photoresist most material factors remain the same for the two wavelengths. The main difference is the number of generated SEs and their energy distribution and diffusion into the matrix that depends on the incident photon energy. Quantitative derivation of this dependence can be simulated using a cellular automaton model ${ }^{31}$, which is beyond the scope of this article and is subject to further investigations. One way to lower $\mathrm{LER}_{\text {mat }}$ is to use alternative development chemistries, but this often comes with the cost of losing sensitivity.

Quantitative evaluation and comparison of the performance of different resists and their dependence on $\lambda$ can be made by a figure of merit. One such rigorous measure is the so-called $K_{\mathrm{LUP}}{ }^{21}$, which has been a standard parameter for assessing the performance of CARs that meet the semiconductor industry's specifications. $K_{\mathrm{LUP}}$ is derived explicitly from the relations between each RLS element with other involved microscopic quantities, including the incident photon energy ${ }^{21}$. The limitation of using such quantity in our evaluation is that that $K_{\text {LUP }}$ can only be used for chemically amplified resists, which have a well defined acid diffusion length. Therefore, in this study we use the so-called Z-parameter for this purpose, since it reveals the explicit relation between macroscopic RLS parameters, is not limited to chemically amplified resists, and has been shown to be an effective and accurate tool for resist evaluation ${ }^{34,35}$. The $Z$ parameter adapted to our conventions reads as follows:

$$
\mathrm{Z}=\mathrm{D}_{0} \times(\mathrm{LER})^{2} \times\left(\mathrm{HP}_{\min }\right)^{3}
$$

where LER corresponds to that of the smallest patterned features with $\mathrm{HP}_{\text {min }}$. Figure 4 illustrates the RLS triangle for all the studied cases. The obtained values at both wavelengths are within the same order of magnitude of other important EUV resists ${ }^{23,34}$. It should be noted that these Z-parameters were evaluated using an IL mask that allowed the printing of line-space structures down to HP $=18 \mathrm{~nm}$ and more compact features were not patterned due to nanofabrication complications that rapidly increase at shorter $P_{\mathrm{g}} \mathrm{s}$. Therefore $\mathrm{HP}_{\text {min }}$ for Inpria IB and HSQ was taken as far as we were able to
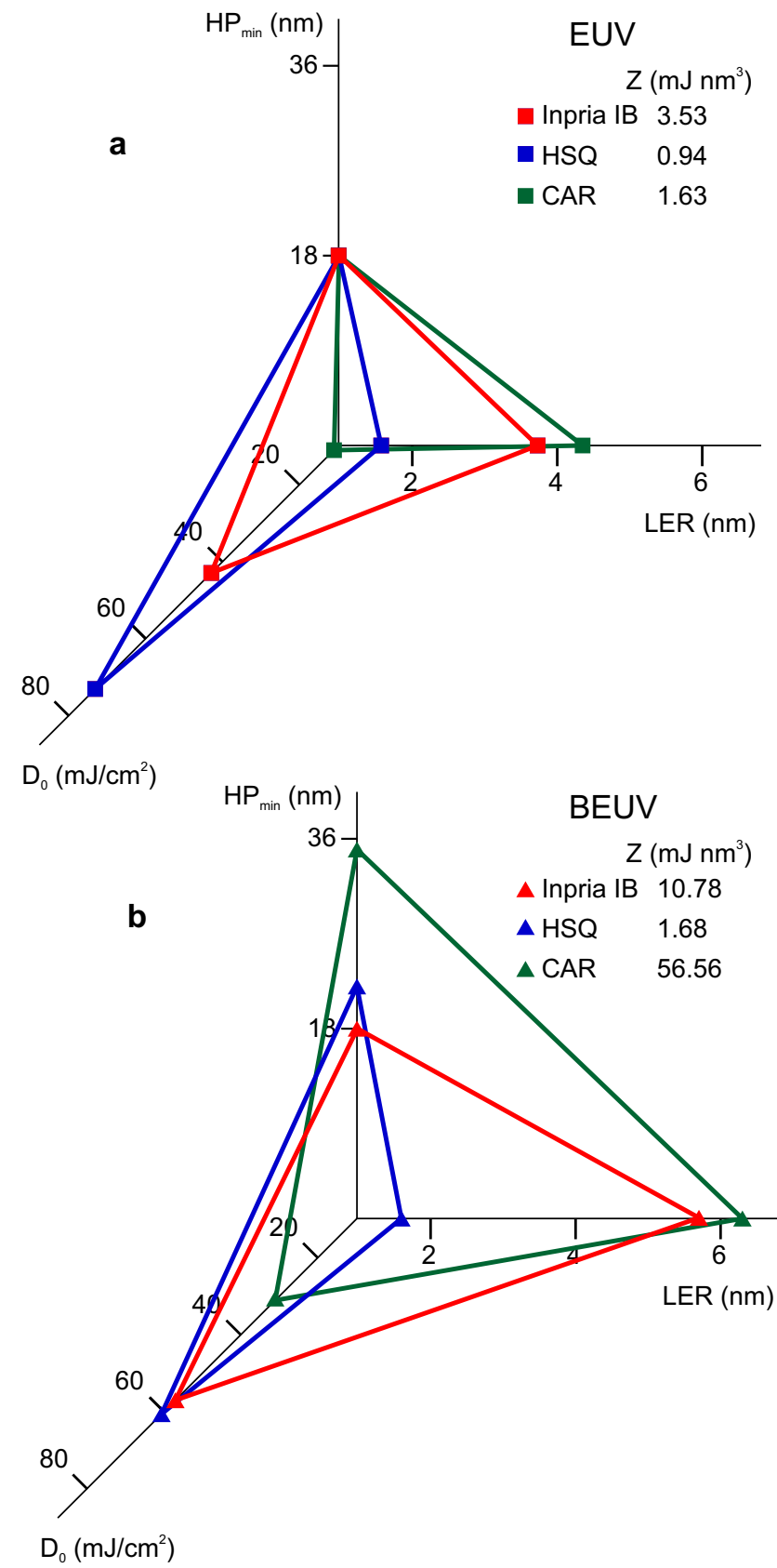

Figure $4 \mid$ Z-parameters. Overall RLS characterization of the photoresists and their evaluated Z-parameters. At (a) EUV and (b) BEUV.

get using this mask. $\mathrm{HP}_{\min }<10 \mathrm{~nm}$ features have been reported for EUV exposures on Inpria IB and $\mathrm{HSQ}^{23,24}$ and therefore finding the exact resolution limits at BEUV requires efficient masks with higher resolution. The very comparable Z-parameter at the two wavelengths for HSQ clearly demonstrates the key role of highly absorbing $\mathrm{Si}$ and suggest using similar elements, such as phosphor and sulfur, for the development of specific BEUV photoresists. As for HSQ itself, the barrier for large-scale utilization of such a resist at BEUV is its worse resolution performance in comparison to EUV, as well as its low sensitivity. It seems that a resist such as Inpria IB would be the best candidate among what has been studied because of a reasonably low Z-parameter at both wavelengths as well as the possibility to provide $<22 \mathrm{~nm}$ resolutions for future technology nodes. For Inpria IB, the main cause of the Z-parameter difference at the two wavelengths is the difference in the LERs, which as explained earlier, can be improved by the reduction of $\mathrm{LER}_{\text {proc }}$ using higher quality mask 
fabrication. When it comes to CAR patterning, the Z-parameter reveals a noticeable difference between the resist performance at the two wavelengths. This is caused by the worse performance of all the three RLS parameters at BEUV, mainly due to the CAR's organic composition, and the acid diffusion process, which amplifies the lower quality of the lines after exposure and development.

In conclusion, lithography at EUV is still the main candidate of high-volume chip manufacturing for the semiconductor industry. Should EUVL reach its single-digit patterning resolution, the choice of using 6.x nm photons for further advancing the resolution requires investments in all aspects of its technology, including light source, optics, and photoresist. Moving to shorter wavelengths for lithography faces the intrinsic increase of LER, caused by shot noise. Moreover, the aerial image quality is more sensitive to defects and roughness in the optics, which also raises the LER. The capability of BEUV photoresist matching the RLS requirements highly depends on their chemical composition. Organic photoresists have very low absorption at BEUV and are unlikely to satisfy sensitivity standards. Using organic CARs to lower the required dose, as we have shown, results in compensation for resolution and LER. It seems that inorganic photoresist using efficiently absorbing elements would be a good choice for reaching future technology nodes of the semiconductor industry with suitable sensitivity and LER. We hope that our findings could pave the way for better understanding and design of novel photoresists that are efficient and functional at photon energy ranges of EUV and beyond.

\section{Methods}

Photoresist parameters. All resists were spin-coated on a 4-inch p-doped Si wafer and were processed as follows: HSQ (Dow Corning ${ }^{\circledR}$ XR-1541) was spin-coated with $5000 \mathrm{rpm}$ for $45 \mathrm{~s}$, which results in resist thickness of $35 \mathrm{~nm}$. After exposure the sample was developed in Tetramethylammonium hydroxide (TMAH) 25\% for $60 \mathrm{~s}$. Inpria (XE15IB) was spin-coated on a wafer treated by oxygen plasma, with $2500 \mathrm{rpm}$ for $45 \mathrm{~s}$, which provides a 20 -nm-thick resist layer. The wafer was then post-apply baked (PAB) at $80^{\circ} \mathrm{C}$ for $120 \mathrm{~s}$ and after exposure post-exposure bake (PEB) with the same temperature and time of PAB. The development is also in TMAH $25 \%$ for $120 \mathrm{~s}$. For CAR, an adhesion underlayer was first prepared and on top, the photoresist was spin-coated with $2500 \mathrm{rpm}$ for $60 \mathrm{~s}$, which provided a 30-nm-thick layer, and was followed by $\mathrm{PAB}$ of $130^{\circ} \mathrm{C}$ for $60 \mathrm{~s}$. PEB was at $110^{\circ} \mathrm{C}$ for $60 \mathrm{~s}$ and the development was in TMAH $0.26 \mathrm{~N}$ for $30 \mathrm{~s}$. The spin-coating of all resists provides a smooth and uniform thin layer and after exposure and development resist thickness loss of $10 \%$, $15 \%$, and $10 \%$ is measured for HSQ, Inpria IB, and the CAR, respectively.

Dose-on-wafer calibration. The $D_{\mathrm{w}}$ calibration was necessary to take into account the effect of the wavelength-dependent diffraction efficiency, $\eta$, of the mask. Experimental evaluation of $\eta$ at each wavelength was done through the process described in reference ${ }^{19}$ by finding the ratio of $D_{0}$ of the first order diffracted beam to the open frame exposure. Since at the patterned area the beam is the sum of the two diffracted beams (Fig. 1a), $D_{\mathrm{w}}$ is given as: $D_{\mathrm{w}}=2 \eta D_{\mathrm{m}}$.

The broadband IL mask. The masks have the benefit of efficiently diffracting light at both EUV and BEUV wavelengths as well as blocking the non-diffracted beams. A broadband mask consists of HSQ line-space gratings and an Au photon stop, made on a free-standing $100 \mathrm{~nm}$ thick $\mathrm{Si}_{3} \mathrm{~N}_{4}$ membrane. On each mask HSQ gratings with different periodicities, in the range of $72<P_{\mathrm{g}}<200 \mathrm{~nm}$ and the height of $\sim 100 \mathrm{~nm}$, are patterned by e-beam lithography. An $8 \mathrm{~nm}$ thick Cr layer is evaporated on $\mathrm{Si}_{3} \mathrm{~N}_{4}$ membrane, prior to spin-coating HSQ, and after fabricating the gratings the $\mathrm{Cr}$ between the grating lines are removed by reactive-ion etching to increase the diffraction efficiency. This $\mathrm{Cr}$ layer serves mainly as a conductive layer during the ebeam lithography step to reduce the sub-field stitching effect. The Au photonstop is $\sim 600 \mathrm{~nm}$ thick and covers the area on the membrane not holding the HSQ gratings. Theoretical calculations and experimental evaluations show that such grating configuration has an IL efficiency of $\sim 8 \%$ at EUV and $\sim 2 \%$ at BEUV. Further Details on the fabrication steps can be found in reference ${ }^{19}$.

1. Tallents, G., Wagenaars, E. \& Pert, G. Optical Lithography at EUV Wavelengths. Nat Photonics 4, 809-811, doi:10.1038/Nphoton.2010.277 (2010).

2. Wagner, C. \& Harned, N. EUV Lithography Lithography Gets Extreme. Nat Photonics 4, 24-26, doi:10.1038/nphoton.2009.251 (2010).

3. Oda, S. \& Ferry, D. Silicon nanoelectronics. (Taylor and Francis, Boca Raton, USA, 2006).

4. Peeters, R. et al. ASML's NXE platform performance and volume introduction. Proc SPIE 8679 doi:10.1117/12.2010932 (2013).
5. Willson, C. G. \& Roman, B. J. The future of lithography: SEMATECH Litho Forum 2008. ACS Nano 2, 1323-1328, doi:10.1021/Nn800410c (2008).

6. Heuberger, A. X-Ray-Lithography. J Vac Sci Technol B 6, 107-121, doi:10.1116/ 1.584026 (1988).

7. EUV Litho, Inc., http://www.euvlitho.com (2010) (Date of access: 01/09/2014).

8. Attwood, D. T. Soft $x$-rays and extreme ultraviolet radiation: principles and applications. (Cambridge university press, Cambridge, England, 2000).

9. O'Sullivan, G. et al. Recent progress in source development for lithography at 6.x nm. Phys Scripta T156, doi:10.1088/0031-8949/2013/T156/014105 (2013).

10. Chkhalo, N. I. et al. High performance La/B4C multilayer mirrors with barrier layers for the next generation lithography. Appl Phys Lett 102, doi:10.1063/ 1.4774298 (2013)

11. Makhotkin, I. A. et al. Spectral properties of La/B - based multilayer mirrors near the boron K absorption edge. Opt Express 20, 11778-11786, doi:10.1364/ OE.20.011778 (2012).

12. Medvedev, V. V. et al. Multilayer mirror with enhanced spectral selectivity for the next generation extreme ultraviolet lithography. Appl Phys Lett 103, doi:10.1063/ 1.4837335 (2013)

13. Tsarfati, T., de Kruijs, R. W. E. V., Zoethout, E., Louis, E. \& Bijkerk, F. Reflective multilayer optics for $6.7 \mathrm{~nm}$ wavelength radiation sources and next generation lithography. Thin Solid Films 518, 1365-1368, doi:10.1016/J.Tsf.2009.09.073 (2009).

14. Oyama, T. G., Oshima, A., Washio, M. \& Tagawa, S. Evaluation of resist sensitivity in extreme ultraviolet/soft x-ray region for next-generation lithography. AIP $A d v$ 1 doi:10.1063/1.3665672 (2011).

15. Oyama, T. G., Oshima, A., Washio, M. \& Tagawa, S. Method of predicting resist sensitivity for 6.x nm extreme ultraviolet lithography. J Vac Sci Technol B 31, doi:10.1116/1.4813789 (2013).

16. Anderson, C. et al. The SEMATECH Berkeley MET: demonstration of 15-nm half-pitch in chemically amplified EUV resist and sensitivity of EUV resists at 6.x$\mathrm{nm}$. Extreme Ultraviolet (EUV) Lithography III 8322, doi:10.1117/12.917386 (2012).

17. Paivanranta, B., Langner, A., Kirk, E., David, C. \& Ekinci, Y. Sub-10 nm patterning using EUV interference lithography. Nanotechnology 22, doi:10.1088/ 0957-4484/22/37/375302 (2011).

18. Solak, H. H. Space-invariant multiple-beam achromatic EUV interference lithography. Microelectron Eng 78, 410-416, doi:10.1016/j.mee.2005.01.012 (2005).

19. Mojarad, N., Fan, D., Gobrecht, J. \& Ekinci, Y. Broadband interference lithography at extreme ultraviolet and soft x-ray wavelengths. Opt Lett 39, 2286-2289, doi:10.1364/Ol.39.002286 (2014).

20. Mojarad, N., Vockenhuber, M., Wang, L., Terhalle, B. \& Ekinci, Y. Patterning at $6.5 \mathrm{~nm}$ wavelength using interference lithography. SPIE Advanced Lithography, 867924-867927, doi:10.1117/12.2011556 (2013).

21. Van Steenwinckel, D., Gronheid, R., Van Roey, F., Willems, P. \& Lammers, J. H. Novel method for characterizing resist performance. J Micro-Nanolith Mem 7, doi:10.1117/1.2909204 (2008).

22. Oleksak, R. P. et al. Chemical and Structural Investigation of High-Resolution Patterning with HafSOx. ACS Appl Mater Inter 6, 2924-2928, doi:10.1021/ am405463u (2014).

23. Ekinci, Y., Vockenhuber, M., Mojarad, N. \& Fan, D. EUV resists towards $11 \mathrm{~nm}$ half-pitch. SPIE Advanced Lithography, 904804-904810, doi:10.1117/12.2046459 (2014).

24. Mojarad, N., Hojeij, M., Wang, L., Gobrecht, J. \& Ekinci, Y. Single-digit-resolution nanopatterning with extreme ultraviolet light for the $2.5 \mathrm{~nm}$ technology node and beyond. Nanoscale (Accepted Manuscript), 7, 4031-4037, doi:10.1039/ C4NR07420C (2015).

25. Grigorescu, A. E. \& Hagen, C. W. Resists for sub-20-nm electron beam lithography with a focus on HSQ: state of the art. Nanotechnology 20, doi:10.1088/ 0957-4484/20/29/292001 (2009).

26. Ekinci, Y., Vockenhuber, M., Hojeij, M., Wang, L. \& Mojarad, N. M. Evaluation of EUV resist performance with interference lithography towards $11 \mathrm{~nm}$ half-pitch and beyond. SPIE Advanced Lithography, 867910-867910-867911, doi:10.1117/ 12.2011533 (2013).

27. Gullikson, E. X-Ray Interactions With Matter, http://henke.lbl.gov/optical_ constants (2010) (Date of access: 01/09/2014).

28. Bae, W. J. et al. High refractive index and high transparency $\mathrm{HfO} 2$ nanocomposites for next generation lithography. J Mater Chem 20, 5186-5189, doi:10.1039/C0jm00679c (2010).

29. Stowers, J. \& Keszler, D. A. High resolution, high sensitivity inorganic resists. Microelectron Eng 86, 730-733, doi:10.1016/J.Mee.2008.11.034 (2009).

30. Kozawa, T. \& Tagawa, S. Radiation Chemistry in Chemically Amplified Resists Jpn J Appl Phys 49, doi:10.1143/Jjap.49.030001 (2010).

31. Brainard, R. L. et al. Shot noise, LER, and quantum efficiency of EUV photoresists. Microlithography 2004, 74-85, doi:10.1117/12.536411 (2004).

32. Levinson, H. J. Principles of lithography. Vol. 146 (SPIE press, Bellingham, USA, 2005).

33. George, S. A. et al. Extreme ultraviolet mask substrate surface roughness effects on lithographic patterning. J Vac Sci Technol B 28, C6e23-C26e30, doi:10.1116/ $1.3502436(2010)$ 
34. Higgins, C. D. et al. Resolution, Line-Edge Roughness, Sensitivity Tradeoff, and Quantum Yield of High Photo Acid Generator Resists for Extreme Ultraviolet Lithography. Jpn J Appl Phys 50, doi:10.1143/Jjap.50.036504 (2011).

35. Wallow, T. et al. Evaluation of EUV resist materials for use at the $32 \mathrm{~nm}$ half-pitch node. Proc SPIE 6921, 69211F, doi:10.1117/12.772943 (2008).

\section{Acknowledgments}

We would like to thank Michaela Vockenhuber for technical support, Sangsul Lee for fruitful discussions, and photoresist suppliers for providing materials. Part of this work was performed at the Swiss Light Source, Paul Scherrer Institute, Switzerland.

\section{Author contributions}

N.M. and Y.E. conceived the experiments. N.M. carried out the mask fabrication, performed the experiments and characterizations, and analyzed the data. Y.E. and J.G. provided expertise on the nanofabrication. N.M. and Y.E. wrote the manuscript. All authors discussed the results and contributed to the final version of the manuscript.

\section{Additional information}

Competing financial interests: The authors declare no competing financial interests.

How to cite this article: Mojarad, N., Gobrecht, J. \& Ekinci, Y. Beyond EUV lithography: a comparative study of efficient photoresists' performance. Sci. Rep. 5, 9235; DOI:10.1038/ srep09235 (2015)

This work is licensed under a Creative Commons Attribution 4.0 International License. The images or other third party material in this article are included in the article's Creative Commons license, unless indicated otherwise in the credit line; if the material is not included under the Creative Commons license, users will need to obtain permission from the license holder in order to reproduce the material. To view a copy of this license, visit http://creativecommons.org/licenses/by/4.0/ 